\title{
IDENTIFICATION OF SOURCES OF RESISTANCE AND BIOCHEMICAL MANAGEMENT OF LATE BLIGHT OF POTATO (PHYTOPHTHORA INFESTANS)
}

\author{
Abdul Rehman Sajid', Ateeq ur Rehman'1, Syed Atif Hasan Naqvi', Ummad ud Din Umar'1, Hameed Ullah Khan \\ Sherwani ${ }^{1}$, Muhammad Rafique Bhatti ${ }^{2}$ \\ ${ }^{1}$ Department of Plant Pathology, Bahauddin Zakariya University, Multan, Pakistan. \\ ${ }^{2}$ FMC Private Limited, Pakistan.
}

\section{ART ICLE IN F O}

\section{Article history}

Received: July 07, 2019

Revised: September 27, 2019

Accepted: December 15, 2019

\section{Keywords}

Soil borne

Vegetable

Late Blight

Phytopthora
A B S T R A C T

\begin{abstract}
A bulk of potato varieties were screened against Phytophthora infestans causing the late blight of potato. Almost all the potato germplasm showed a varying response against the devastating pathogen at Okara and Sahiwal. Field trials were consisted of screening of germplasm, area under disease progress curve and chemical management of the disease by using the various fungicides. Randomized complete block design was used for the research at both locations. Out of 68 genotypes/ varieties 16 were susceptible, 20 moderately resistant, five highly susceptible and 27 as moderately susceptible. Maximum disease incidence was recorded on Desiree and Cardinal (90\%) followed by Santee (83.33\%). The maximum mean disease severity of 9.48 was noted on Cardinal followed by Desiree 9.11 and Karoda 9.0 with the area under disease progress curve 28.55, 27.33 and 26.00 respectively. The results showed that $5 \mathrm{gm} / \mathrm{L}$ was the best dose against the suppression and control of the potato late blight under the field conditions. Azoxystrobin which is a new chemistry fungicide found to be the best chemical treatment against the late blight of potato followed by the copper oxychloride-based fungicides Cobox which also showed the best response. Aliette, Antracol, Tebuconazole and Mancozeb were also played a role to minimize the disease yet their higher concentration was even not so effective to combat the disease. Mancozeb was not found to be effective to control the disease as compared to the other three fungicides and orange oil extract.
\end{abstract}

Corresponding Author: Syed Atif Hasan Naqvi

Email: atifhasanshah@hotmail.com

(C) 2019 EScience Press. All rights reserved.

\section{INTRODUCTION}

Potato (Solanum tuberosum L.) is the world's most significant and leading vegetable crop which provides remunerative income due to its inherent bulk production potential along with nutrition. Pakistan is blessed with the favorable climatic and edaphic conditions ideal for potato production. It is the fourth major crop of Pakistan after wheat, rice and maize. In
Pakistan, potato was cultivated on about 3,000 hectares at the time of independence which increased to 179,300 hectares with a production of $3,849.5 \mathrm{t} / \mathrm{h}$. Fresh potato crop contains moisture, dry matter and starch at the rate of 79, 21 and 60-80\%, respectively (FAO, 2017). Potato yield is influenced mainly by temperature during growing season. In early stages of potato crop, a temperature of $24^{\circ} \mathrm{C}$ is optimum and in later growth 
stage, $18^{\circ} \mathrm{C}$ is most suitable. Maximum tubers are produced at $20^{\circ} \mathrm{C}$, and when temperature rises production decreases and stops at $30^{\circ} \mathrm{C}$. Tuber production is favored by short days. Although, Pakistan has favorable set of environmental conditions for potato production but the average yield per hectare is very low when compared to other countries (Kelman, 1984).

Diseases are the most lethal factor for low production of potato in Pakistan. In Pakistan, potato is attacked by a number of diseases caused by different pathogens like bacteria, fungi and viruses. Late blight of potato (Phytophthora infestans, Mont. De Bary) is an important disease of potato which causes heavy yield losses in Pakistan. An oomycete family member, P. infestans is the cause of this devastating disease of potato. The disease was first documented in North America and Europe in the middle of $19^{\text {th }}$ century and caused many devastating epidemics including famous Irish potato famine of 18451846 (Fry et al., 1993). Late blight is a polycyclic disease which affects both foliar parts and tubers. Even though the pathogen has a complex life cycle which consists of both aerial and soil phases, it is most often viewed as a typical, aerial pathogen with prolific production of short lived asexual infective sporangia (Hall et al., 2004). Under conducive climatic conditions, it takes only two days to destroy potato field. Late blight association with secondary pathogens and soft rotting bacteria can lead to severe disease of tuber blight (2-5\%) that can lead to complete loss of potatoes in storage (Dalsgaard and Pedersen, 1996). The disease occurs with great severity in the hilly areas of Pakistan especially in Kaghan valley. In 2006-07, the disease appeared in almost all the potato growing areas as a major disease. Conducive conditions for sever disease are temperature ranging from 10 to $25^{\circ} \mathrm{C}$ plus heavy rain or dew. Characteristic symptoms of the disease are brown spots surrounded by chlorotic haloes on leaves. Similarly, during the moist weather, white cottony growth appears along the margins of lesions on the lower side of the leaves. Petioles and infected stems turn black or dark brown. Lesions produced on leaves and stems further serve as source of inoculum. Spores from the leaf and stem lesions are transported downward where they infect tubers by leaching down through soil profile. On tubers, these spores germinate and produce brown to purple lesions which result in brown rot. Due to rot and blight of potato tubers, serious losses of marketable yield occurred. After harvest, some of the blighted tubers remain in the field which serve as inoculum for the next crop as P. infestans has the capability to survive in the soil for several years as thick walled sexual oospores. These sexual spores when germinate give rise to asexual spores called sporangia, which survive only on living host such as cull potatoes. Sporangia disseminate by rain splashes and wind. When temperature increases above $10^{\circ} \mathrm{C}$, spores germinate and infect leaf directly whereas when temperature is low, these sporangia produce motile zoospores (10-12) which can infect leaves. P. infestans overwinters in infected tubers of previous season as mycelium. In spring, when temperature increases which favors tuber to grow, infected tubers produce infected plant, as mycelium within it grows to produce infected plant on which spores bearing lesions are produced. Newly planted crop is infected by these spores and the disease cycle continues (Ghorbani et al., 2004).

Cultivation of late blight resistant varieties is the best economical solution to protect this devastating disease. For this purpose, screening of commercially available potato germplasm is necessary. As late blight of potato is of great economic importance because of its wide distribution in potato producing areas of the Pakistan. The present studies were designed with the objective to screen potato cultivars for resistance against the disease under natural environmental conditions and to manage the disease with some broad-spectrum fungicides and orange oil extract.

\section{MATERIALS AND METHODS}

Study site: The experiments for the evaluation of resistance sources of potato against late blight and its bio-chemical management were conducted at two different locations i.e. Sahiwal $\left(30.6644^{\circ}\right.$ N, $73.1083^{\circ}$ E, $152 \mathrm{~m}$ from the sea level) and Okara $\left(30.8091^{\circ} \mathrm{N}\right.$, $73.4477^{\circ} \mathrm{E}, 105 \mathrm{~m}$ from the sea level).

Plant material for evaluation of germplasm resistance: The experiment was conducted in a randomized complete block design with three replicates. Tubers of various genotypes of potato were provided by Bayer Crop Sciences, Pakistan. Seeds of these 68 varieties/lines viz. Lady rosetta, Ajax, Patrones, Santee, Lala faisal, Desiree, Symponia, Hermes, Multa, Oscar, Ultimas, FD 51-6, FD 71-1, FD 1-10, FD 65-6, FD 52-2, N22, SH-788, FD 49-62, Shanan, NO. 9619, FD 63-2, N34, FSD White, Siply red, Rodio, Marato, SH-692, Cardinal, FD 56-1, FD 70-1, SH-692, FD 35-25, FD 1-3, FD 53-7, FD 32-2, FD 8-3, FD 3-9, FD 6901, FD 35-36, FD 632, Karoda, FD 37-13, FD 51-5, FD 52-2, FD 63-4, N-8, FD 
48-54, N-30, FD 64-2, FD 3-10, FD 61-3, N-13, FD 49-62, FD 51-5, FD 8-1, FD 61-3, FD 1-10, FD 53-6, FD 65-4, SH704, N-37, N39-6, N-18, SH 216 A, SH-5, FSD Red, FD 7659 were sown on ridges and allowed to grow with routinely cultural practices. Field layout comprised a 15 $\mathrm{cm}$ plant to plant and $60 \mathrm{~cm}$ row to row distance. Each replication comprised of three rows with 7 plants in each row. A line of highly susceptible variety was planted after every three varieties as disease spreader. The whole research area was encompassed by two rows of spreader to promote maximum disease pressure. Normal cultural and agronomic practices were adopted to maintain crop in healthy condition.

Assessment of disease severity, incidence and AUDPC: Disease severity was evaluated according to the scale given by Shutong et al. (2007) after thirty days of planting as the first symptoms appeared on the plants. The scale for the assessment of the disease severity is given in Table 1.

Disease incidence and the disease severity were calculated by the following formulae:

Disease incidence (\%)of late blight of potato

$$
=\frac{\text { No of infected plants }}{\text { Total number of plants }} \times 100
$$

Disease severity

$=\frac{D S L \times \text { No. of leaflets with same DSL }}{\text { Total number of leaflets } \times 9} \times 100$

Where DSL $=$ Disease Severity Level

While AUDPC was calculated by following formula:

$$
\text { AUDPC }=\sum_{i=1}^{n-1}\left[\frac{\mathrm{X} i+\mathrm{Xi}+1}{2}\right](t i+1-t i)
$$

where, $n$ shows disease rating dates, $X i$ show the specific date for data collection, and $\left(t_{i+1}-t_{i}\right)$, interval between two disease rating dates

Table 1 . The scale for the assessment of the disease severity.

\begin{tabular}{cl}
\hline Rating & Description \\
\hline 0 & No disease \\
1 & Small lesion on the inoculated point with the lesion arealess than $10 \%$ of the whole leaflet \\
3 & Lesion area between 10 and $20 \%$ of whole leaflet \\
5 & $\begin{array}{l}\text { Lesion area between } 20 \text { and } 30 \% \text { of whole leaflet, the waterish area less than } 50 \% \text { of the whole } \\
\text { leaflet }\end{array}$ \\
7 & Lesion area between 30 and $60 \%$ \\
9 & Lesion area over $60 \%$ of the whole leaflet
\end{tabular}

Evaluation of various fungicides and botanical oils against late blight of potato: The effectiveness of fungicides and botanical oils (Table 2) was tested against the disease using three susceptible varieties viz. Desiree, Sante and Cardinal. The varieties were sown at the same experimental area.

Test fungicides viz. Aliette, Antracol, Tebuconazole, Copper oxychloride, Mancozeb and Azaxystorulubins (Table 2) were applied at the rate of $1 \mathrm{~g} / \mathrm{liter}, 3 \mathrm{~g} /$ liter and $5 \mathrm{~g} /$ liter by hand sprayer consisting of a boom with two XR11003VS flat fan nozzles at regular intervals of 12 days. Each treatment was replicated thrice while for the control no fungicide was applied.

Similarly, the test botanical oils viz. neem, clove and mint (Table 1) were collected from the local market and diluted in hexane (organic solvent) and applied at the rate of $1 \mathrm{~g}$ / lietr, $3 \mathrm{~g}$ / liter and $5 \mathrm{~g} /$ liter by hand sprayer consisting of a boom with two XR11003VS flat fan nozzles at regular intervals of 12 days. All the treatments were completely randomized.

Statistical analysis: All the collected data were statistically analyzed and subjected to analysis of variance (ANOVA). Treatments means of all data were compared by the Fisher's least significant difference (LSD) (Steel et al., 1980) test at $(\mathrm{P} \leq 0.05)$ by using the statistical software package.

\section{RESULTS}

In vivo studies for late blight of potato through screening of germplasm: Analysis of Duncan's multiple range test indicated significant $(P<0.05)$ variation among all the varieties/germplasm for potato late blight in the natural environment. Disease incidence and severity data of 68 potato varieties against potato late blight at different locations viz. Okara and Sahiwal showed statistically significant difference in mean disease incidence and severity at $5 \%$ level of significance. Out of 68 genotypes/varieties at Okara, the incidence showed 16 varieties as 
susceptible, 20 varieties as moderately resistant, five varieties as highly susceptible and 27 varieties as moderately susceptible. The maximum disease incidence at Okara was recorded at Desiree and Cardinal (90\%) followed by Santee (83.33\%), whereas minimum disease incidence was noted on SH-5 (26.67\%). Similarly, at Sahiwal area 16 genotypes were found susceptible, 36 as moderately susceptible, six varieties as highly susceptible and 10 varieties as moderately resistant. Maximum mean disease incidence at Sahiwal was noted on Cardinal and Desiree (91.67\%) and Karoda and Santee (90\%) while minimum disease incidence was noted $33.33 \%$ on $\mathrm{SH}$ 216 A, N13, FD 1-3 and FD 1-10. The disease severity data at Okara area showed 34 genotypes as susceptible, 10 genotypes as moderately susceptible, 14 genotypes as moderately resistant and 10 genotypes as highly susceptible. The maximum mean disease severity 9.48 was noted on Cardinal, followed by Desiree 9.11 and Karoda 9.0 with the area under disease progress curve $28.55,27.33$ and 26.00 respectively while minimum disease severity was recorded 3.91 on Multa and 4.41 on FD 56-1 with the area under disease progress curve 11.33 and 13.33 respectively (Table 3 ).

Table 2. List of fungicides and botanicals oils evaluated against potato late blight.

\begin{tabular}{cllcl}
\hline \multirow{2}{*}{ Sr. No. } & \multicolumn{5}{c}{ Fungicides } \\
\cline { 2 - 5 } & Name & Active ingredient & Form available & Company \\
\hline 1. & Aliettee & Focetyl Aluminium & $250 \mathrm{FS}$ & Bayer Crop Sciences \\
2. & Antracol & Propineb & $50 \mathrm{WP}$ & Arysta Life Sciences \\
3. & Tegula & Tebuconazole & $10 \mathrm{SL}$ & Bayer Crop Sciences \\
4. & Cobox & Copper oxychloride & $70 \mathrm{WP}$ & FMC \\
5. & Dithane M-45 & Mancozeb & $70 \mathrm{WP}$ & Bayer Crop Sciences \\
6. & Azaxystorulubins & Azaxystorulubins & $250 \mathrm{EC}$ & AgPharma \\
\hline \multicolumn{5}{c}{ Botanicals oils } \\
\hline 7 & Neem & Azadiractin & Oil dissolved in solvent & Local Market \\
9 & Clove & Eugenol & Oil dissolved in solvent & \\
\hline
\end{tabular}

*FS= Flowable slurry, WP= Wettable powder, $\mathrm{SL}=$ Soluable liquid, EC= Emulsifiable concentrate

Table 3. Disease incidence, severity and area under disease progress curve of potato germplasm against late blight at Okara and Sahiwal.

\begin{tabular}{|c|c|c|c|c|c|}
\hline \multirow{2}{*}{$\begin{array}{l}\text { Sr. } \\
\text { No. }\end{array}$} & \multirow[t]{2}{*}{ Variety } & $\begin{array}{l}\text { Disease incidence } \\
\text { (\%) \& Response }\end{array}$ & $\begin{array}{l}\text { Disease incidence } \\
\text { (\%) \& Response }\end{array}$ & $\begin{array}{c}\text { Disease severity, } \\
\text { Response \& [AUDPC] }\end{array}$ & $\begin{array}{c}\text { Disease severity, } \\
\text { Response \& [AUDPC] }\end{array}$ \\
\hline & & At Okara & At Sahiwal & At Okara & At Sahiwal \\
\hline 1 & Lady rosetta, & $70.00 \mathrm{bc}(\mathrm{S})$ & 66.67 c-e (S) & 7.33 c-e (S) ${ }^{[21.66]}$ & 6.99 e-l (S) ${ }^{[20.88]}$ \\
\hline 2 & Ajax, & $70.00 \mathrm{bc}(\mathrm{S})$ & $73.33 \mathrm{bc}(\mathrm{HS})$ & 5.18 i-p (MR) ${ }^{[17.66]}$ & 4.85 o-q (MR) ${ }^{[12.66]}$ \\
\hline 3 & Patrones, & $60.00 \mathrm{c}-\mathrm{f}(\mathrm{S})$ & 60.00 c-g (MS) & 6.66 c-j (S) ${ }^{[19.44]}$ & 7.10 f-n (S) ${ }^{[20.99]}$ \\
\hline 4 & Santee, & 83.33 a (HS) & 90.00 a (HS) & $9.10 \mathrm{ab}(\mathrm{HS})[27.33]$ & $9.44 \mathrm{ab}(\mathrm{HS})$ [27.33] \\
\hline 5 & Lala faisal, & $80.00 \mathrm{ab}$ (HS) & $83.33 \mathrm{ab}(\mathrm{HS})$ & $7.10 \mathrm{c}-\mathrm{f}(\mathrm{S})^{[20.11]}$ & 7.44 d-h (S) ${ }^{[20.11]}$ \\
\hline 6 & Desiree, & 90.00 a (HS) & 91.67 a (HS) & 9.11 a (HS) [27.73] & 9.66 a (HS) ${ }^{[28.55]}$ \\
\hline 7 & Symponia, & $50.00 \mathrm{fg}(\mathrm{MS})$ & 46.67 g-k (MS) & $5.07 \mathrm{k}-\mathrm{p}(\mathrm{MS})^{[14.66]}$ & 5.59 i-p (MS) [18.60] \\
\hline 8 & Hermes, & $50.00 \mathrm{fg}(\mathrm{MS})$ & 46.67 g-k (MS) & 4.33 n-p (MR) [12.44] & $5.52 \mathrm{l}-\mathrm{q}(\mathrm{MS}){ }^{[12.44]}$ \\
\hline 9 & Multa, & $70.00 \mathrm{bc}(\mathrm{S})$ & 63.33 c-e (S) & 3.92 p (MR) [11.33] & $4.92 \mathrm{o-q}(\mathrm{MR})^{[11.33]}$ \\
\hline 10 & Oscar, & 63.33 c-e (S) & $56.67 \mathrm{~d}-\mathrm{h}(\mathrm{MS})$ & $6.59 \mathrm{c}-\mathrm{f}(\mathrm{S})^{[20.33]}$ & 7.37 d-h (S) ${ }^{[20.33]}$ \\
\hline 11 & Ultimas, & $60.00 \mathrm{c}-\mathrm{f}(\mathrm{S})$ & $70.00 \mathrm{~b}-\mathrm{d}(\mathrm{S})$ & 5.77 f-n (MS) [18.66] & 6.25 i-h (S) ${ }^{[18.66]}$ \\
\hline 12 & FD 51-6, & $50.00 \mathrm{fg}(\mathrm{MS})$ & 46.67 g-k (MS) & 4.55 n-p (MR) [13.33] & 4.37 r (MR) ${ }^{[13.33]}$ \\
\hline 13 & FD 71-1, & $40.00 \mathrm{gh}(\mathrm{MR})$ & $36.67 \mathrm{kl}(\mathrm{MR})$ & 6.33 d-m (S) [20.99] & 5.78 i-p (MS) [19.01] \\
\hline 14 & FD 1-10, & 36.67 hi (MR) & 33.33 (MR) & $6.97 \mathrm{c}-\mathrm{g}(\mathrm{S})^{[21.89]}$ & 6.86 e-l (S) ${ }^{[19.44]}$ \\
\hline
\end{tabular}




\begin{tabular}{|c|c|c|c|c|c|}
\hline 15 & FD 65-6, & 56.67 d-f (MS) & 53.33 e-i (MS) & $4.77 \mathrm{~m}-\mathrm{o}(\mathrm{MR}){ }^{[13.22]}$ & 5.88 i-q (MS) [19.01] \\
\hline 16 & FD 52-2, & 53.33 ef (MS) & 46.67 g-k (MS) & $6.51 \mathrm{c}-\mathrm{f}(\mathrm{S})^{[20.00]}$ & $6.58 \mathrm{i}-\mathrm{r}(\mathrm{S})^{[21.01]}$ \\
\hline 17 & N22, & 63.33 c-e (S) & 53.33 e-i (S) & 5.56 g-n (MS) ${ }^{[18.60]}$ & $6.08 \mathrm{i}-\mathrm{m}(\mathrm{S})^{[20.36]}$ \\
\hline 18 & SH-788, & $50.00 \mathrm{fg}(\mathrm{MS})$ & $56.67 \mathrm{~d}-\mathrm{h}(\mathrm{S})$ & 5.75 f-n (MS) ${ }^{[19.01]}$ & $6.15 \mathrm{k}-\mathrm{m}(\mathrm{S})^{[19.01]}$ \\
\hline 19 & FD 49-62, & 53.33 ef (MS) & $50.00 \mathrm{f}-\mathrm{i}(\mathrm{MS})$ & $5.48 \mathrm{i}-\mathrm{p}(\mathrm{MS})^{[16.66]}$ & $5.48 \mathrm{l}-\mathrm{q}(\mathrm{MS})^{[16.66]}$ \\
\hline 20 & Shanan, & 60.00 c-f (MS) & 53.33 e-i (MS) & $8.99 \mathrm{ab}(\mathrm{HS})$ [25.99] & 8.33 a-c (HS) [25.99] \\
\hline 21 & No. 9619, & $60.00 \mathrm{c}-\mathrm{f}(\mathrm{MS})$ & $63.33 \mathrm{c}-\mathrm{f}(\mathrm{S})$ & 7.12 e-g (S) ${ }^{[20.99]}$ & 7.20 e-j (S) [20.99] \\
\hline 22 & FD 63-2, & 69.00 b-d (S) & $60.00 \mathrm{c}-\mathrm{g}(\mathrm{S})$ & $6.40(\mathrm{~S})^{[19.11]}$ & $6.48 \mathrm{f}-\mathrm{n}(\mathrm{S})^{[19.11]}$ \\
\hline 23 & $\mathrm{~N}-34$ & $40.00 \mathrm{gh}(\mathrm{MR})$ & 46.67 g-k (MS) & $4.30 \mathrm{n}-\mathrm{p}(\mathrm{MR}){ }^{[13.01]}$ & $4.74 \mathrm{qr}(\mathrm{MR})^{[13.01]}$ \\
\hline 24 & FSD White, & $66.67 \mathrm{~cd}(\mathrm{~S})$ & 56.67 d-h (MS) & $6.39 \mathrm{i}-\mathrm{m}(\mathrm{S})^{[21.00]}$ & $6.47 \mathrm{f}-\mathrm{n}(\mathrm{S})^{[21.00]}$ \\
\hline 25 & Siply red, & $50.00 \mathrm{fg}(\mathrm{MS})$ & 53.33 e-i (MS) & $6.45 \mathrm{c}-\mathrm{f}(\mathrm{S})^{[21.11]}$ & $6.52 \mathrm{i}-\mathrm{n}(\mathrm{S})^{[21.11]}$ \\
\hline 26 & Rodio, & 60.00 c-f (MS) & $60.00 \mathrm{c}-\mathrm{g}(\mathrm{S})$ & 7.59 b-d (HS) ${ }^{[22.66]}$ & 7.92 b-f (HS) [22.66] \\
\hline 27 & Marato, & $40.00 \mathrm{gh}(\mathrm{MR})$ & 46.67 g-k (MS) & $9.00 \mathrm{ab}(\mathrm{HS})^{[26.00]}$ & $9.00 \mathrm{a}-\mathrm{d}(\mathrm{HS})^{[27.00]}$ \\
\hline 28 & SH-692, & 56.67 d-f (MS) & 53.33 e-i $(S)$ & 4.56 n-p (MR) ${ }^{[12.66]}$ & 5.04 o-q (MR) ${ }^{[16.66]}$ \\
\hline 29 & Cardinal, & 90.00 a (HS) & 91.67 a (HS) & 9.48 a (HS) ${ }^{[28.55]}$ & $9.59 \mathrm{a}(\mathrm{HS})^{[28.55]}$ \\
\hline 30 & FD 56-1, & 56.67 d-f (MS) & 53.33 f-i (MS) & $4.41 \mathrm{n}-\mathrm{p}(\mathrm{MR})$ [13.33] & 4.82 p-r (MR) [12.92] \\
\hline 31 & FD 70-1, & $50.00 \mathrm{fg}(\mathrm{MS})$ & $50.00 \mathrm{f}-\mathrm{i}(\mathrm{MS})$ & 5.67 f-n (MS) [18.67] & $6.11 \mathrm{i}-\mathrm{q}(\mathrm{S})^{[18.67]}$ \\
\hline 32 & SH-692, & $50.00 \mathrm{fg}(\mathrm{MS})$ & 53.33 e-i (MS) & 7.89 a-d (HS) [24.11] & 7.93 c-f (HS) [24.11] \\
\hline 33 & FD 35-25, & $40.00 \mathrm{gh}(\mathrm{MR})$ & $36.67 \mathrm{kl}(\mathrm{MR})$ & $6.45 \mathrm{c}-\mathrm{j}(\mathrm{S})^{[20.06]}$ & 6.85 e-l (S) ${ }^{[20.06]}$ \\
\hline 34 & FD 1-3, & $40.00 \mathrm{gh}(\mathrm{MR})$ & 33.33 kl (MR) & $6.78 \mathrm{c}-\mathrm{h}(\mathrm{S})^{[21.01]}$ & $7.19 \mathrm{f}-\mathrm{k}(\mathrm{S})^{[21.01]}$ \\
\hline 35 & FD 53-7, & $40.00 \mathrm{gh}(\mathrm{MR})$ & $30.00 \mathrm{kl}(\mathrm{MR})$ & 4.93 k-p (MR) ${ }^{[14.00]}$ & $5.41 \mathrm{o}-\mathrm{q}(\mathrm{MS})^{[15.78]}$ \\
\hline 36 & FD 32-2, & $50.00 \mathrm{fg}(\mathrm{MS})$ & 50.00 f-i (MS) & $6.41 \mathrm{c}-\mathrm{l}(\mathrm{S})^{[21.04]}$ & 6.48 i-p (S) [19.22] \\
\hline 37 & FD 8-3, & 63.33 c-e (S) & 56.67 d-h (MS) & 6.77 c-h (S) ${ }^{[21.01]}$ & $7.15 \mathrm{f}-\mathrm{k}(\mathrm{S}){ }^{[21.01]}$ \\
\hline 38 & FD 3-9, & $50.00 \mathrm{fg}(\mathrm{MS})$ & 50.00 f-i (MS) & 7.15 e-g (S) ${ }^{[21.00]}$ & 7.60 ce $(S)^{[21.00]}$ \\
\hline 39 & FD 6901, & $60.00 \mathrm{c}-\mathrm{f}(\mathrm{S})$ & 56.67 d-h (MS) & $6.79 \mathrm{c}-\mathrm{h}(\mathrm{S})^{[21.01]}$ & 7.30 d-h (S) [21.01] \\
\hline 40 & FD 35-36, & $70.00 \mathrm{bc}(\mathrm{S})$ & 66.67 c-e (S) & $5.67 \mathrm{i}-\mathrm{m}(\mathrm{MS})^{[18.66]}$ & 6.15 i-m (S) [18.40] \\
\hline 41 & FD 63-2, & 30.00 hi (MR) & $33.33 \mathrm{kl}(\mathrm{MS})$ & 5.67 f-m (MS) [18.66] & $6.11 \mathrm{i}-\mathrm{q}(\mathrm{S})^{[18.33]}$ \\
\hline 42 & Karoda, & 86.67 a (HS) & 90.00 a (HS) & $9.00 \mathrm{ab}(\mathrm{HS})[26.00]$ & $9.22 \mathrm{a}-\mathrm{d}(\mathrm{HS})$ [27.00] \\
\hline 43 & FD 37-13, & $50.00 \mathrm{fg}(\mathrm{MS})$ & 56.67 d-h (MS) & 6.66 c-j (S) ${ }^{[20.33]}$ & 6.77 i-l (HS) [20.66] \\
\hline 44 & FD 51-5. & 50.00 fg (MS) & 60.00 c-g (MS) & 5.59 g-n (S) ${ }^{[19.33]}$ & 6.07 i-l (HS) [19.88] \\
\hline 45 & FD 52-2, & $60.00 \mathrm{c}-\mathrm{f}(\mathrm{S})$ & $63.33 \mathrm{c}-\mathrm{f}(\mathrm{S})$ & $4.22 \mathrm{p}(\mathrm{MR}){ }^{[13.01]}$ & $4.70 \operatorname{qr}(\mathrm{MR}){ }^{[12.34]}$ \\
\hline 46 & FD 63-4, & $50.00 \mathrm{fg}(\mathrm{MS})$ & 53.33 e-i (MS) & $6.82 \mathrm{c}-\mathrm{g}(\mathrm{S})^{[21.01]}$ & $7.23 \mathrm{i}-\mathrm{h}(\mathrm{S})^{[21.34]}$ \\
\hline 47 & $\mathrm{~N}-8$ & $40.00 \mathrm{gh}(\mathrm{MR})$ & 43.33 h-l (MS) & $6.78 \mathrm{c}-\mathrm{f}(\mathrm{S})^{[21.01]}$ & 6.85 e-l (S) ${ }^{[20.34]}$ \\
\hline 48 & FD 48-54, & 30.00 hi (MR) & $36.67 \mathrm{kl}$ (MS) & 8.03 a-c (HS) [24.01] & 8.35 a-c (HS) [24.34] \\
\hline 49 & $\mathrm{~N}-30$ & $50.00 \mathrm{fg}(\mathrm{MS})$ & 53.33 e-i (MS) & $6.63 \mathrm{c}-\mathrm{f}(\mathrm{S})^{[20.36]}$ & 7.07 e-k (S) ${ }^{[20.03]}$ \\
\hline 50 & FD 64-2, & 60.00 c-f (MS) & 66.67 c-e (S) & 6.70 c-n (S) ${ }^{[20.33]}$ & $6.11 \mathrm{i}-\mathrm{p}(\mathrm{S})^{[19.89]}$ \\
\hline 51 & FD 3-10, & 53.33 ef (MS) & $60.00 \mathrm{c}-\mathrm{g}(\mathrm{S})$ & $6.78 \mathrm{c}-\mathrm{h}(\mathrm{S})^{[21.00]}$ & $6.19 \mathrm{i}-\mathrm{p}(\mathrm{S})^{[21.33]}$ \\
\hline 52 & FD 61-3, & 33.33 hi (MR) & $36.67 \mathrm{kl}(\mathrm{MR})$ & 7.89 a-d (HS) [23.66] & $6.93 \mathrm{e}-\mathrm{l}(\mathrm{S})^{[23.89]}$ \\
\hline 53 & $\mathrm{~N}-13$ & 30.00 hi (MR) & $33.33 \mathrm{kl}(\mathrm{MR})$ & 7.77 b-d (HS) [22.02] & 6.85 e-p (S) ${ }^{[22.35]}$ \\
\hline 54 & FD 49-62, & 33.33 hi (MR) & 40.00 i-l (MR) & $6.44 c-1(S)^{[20.66]}$ & $6.11 \mathrm{i}-\mathrm{q}(\mathrm{S})^{[20.22]}$ \\
\hline 55 & FD 51-5, & 33.33 hi (MR) & 40.00 i-l (MR) & 7.52 b-d (S) ${ }^{[20.97]}$ & 6.89 e-l (S) ${ }^{[21.30]}$ \\
\hline 56 & FD 8-1, & $50.00 \mathrm{fg}(\mathrm{MS})$ & 56.67 d-h (MS) & $5.67 \mathrm{i}-\mathrm{n}(\mathrm{MS})^{[18.66]}$ & 5.78 i-q (MS) [18.77] \\
\hline 57 & FD 61-3, & $50.00 \mathrm{fg}(\mathrm{MS})$ & 53.33 e-i (MS) & 4.82 l-p (MR) ${ }^{[13.66]}$ & 4.90 o-q (MR) [13.11] \\
\hline 58 & FD 1-10, & $60.00 \mathrm{c}-\mathrm{f}(\mathrm{S})$ & $63.33 \mathrm{c}-\mathrm{f}(\mathrm{S})$ & $7.21 \mathrm{c}-\mathrm{f}(\mathrm{S})^{[21.33]}$ & $6.81 \mathrm{i}-1(\mathrm{~S})[21.55]$ \\
\hline 59 & FD 53-6, & $50.00 \mathrm{fg}(\mathrm{MS})$ & 56.67 d-h (MS) & $5.89 \mathrm{i}-\mathrm{l}(\mathrm{MS})$ [19.25] & 5.93 k-h (S) ${ }^{[19.37]}$ \\
\hline 60 & FD 65-4, & $50.00 \mathrm{fg}(\mathrm{MS})$ & 56.67 d-h (MS) & $6.91 \mathrm{c}-\mathrm{g}(\mathrm{S})^{[21.77]}$ & $6.99 \mathrm{e}-\mathrm{l}(\mathrm{S})^{[22.00]}$ \\
\hline 61 & SH-704, & $60.00 \mathrm{cf}(\mathrm{MS})$ & 63.33 c-f (MS) & $7.26 \mathrm{c}-\mathrm{f}(\mathrm{S})^{[20.05]}$ & 7.07 e-l (S) ${ }^{[21.33]}$ \\
\hline
\end{tabular}




\begin{tabular}{|c|c|c|c|c|c|}
\hline 62 & $\mathrm{~N}-37$, & 63.33 c-e (S) & 60.00 c-g (S) & 7.07 c-h (S) [19.99] & 6.85 e-l (S) ${ }^{[19.99]}$ \\
\hline 63 & N39-6, & 40.00 gh (MR) & $36.67 \mathrm{kl}(\mathrm{MS})$ & $6.41 \mathrm{c}-\mathrm{m}(\mathrm{S})^{[20.36]}$ & 5.82 i-r (MS) [20.03] \\
\hline 64 & N-18, & 36.67 hi (MR) & $36.67 \mathrm{kl}(\mathrm{MS})$ & 6.66 c-j (S) [20.33] & 7.40 d-h (S) [20.55] \\
\hline 65 & SH 216 A, & 30.00 hi (MR) & $33.33 \mathrm{kl}(\mathrm{MS})$ & 7.24 c-f (S) ${ }^{[20.99]}$ & $6.67 \mathrm{i}-1 \mathrm{~S}(\mathrm{~S})^{[21.33]}$ \\
\hline 66 & SH-5, & 26.67 hi (MR) & $40.00 \mathrm{i}-\mathrm{l}(\mathrm{MR})$ & $6.41 \mathrm{c}-\mathrm{m}(\mathrm{S})^{[19.00]}$ & $6.48 \mathrm{f}-\mathrm{n}(\mathrm{S}){ }^{[19.33]}$ \\
\hline 67 & FSD Red, & 30.00 hi (MR) & 43.33 h-l (MS) & $6.82 \mathrm{c}-\mathrm{h}(\mathrm{S}){ }^{[21.44]}$ & $6.63 \mathrm{i}-\mathrm{k}(\mathrm{S})[21.67]$ \\
\hline 68 & FD 76-59, & $70.00 \mathrm{bc}(\mathrm{S})$ & 66.67 d-h (S) & $7.33 \mathrm{c}-\mathrm{f}(\mathrm{S})^{[21.66]}$ & $6.99 \mathrm{i}-1 \mathrm{SS})^{[20.88]}$ \\
\hline
\end{tabular}

${ }^{*}$ Means followed by the same letter in each column are not statistically different at (0.05); AUDPC= area under progress curve, Values in parenthesis are the AUDPC values of each variety, while Letters in parenthesis are the response of each variety towards the disease.

Bio-chemical management of late blight of potato: Bio-chemical management of the disease using some fungicides and orange oil extract against the late blight of potato showed significant reduction in disease severity as compared to control at all the tested concentrations which were same both for fungicides and oil extract. The results showed that $5 \mathrm{~g} / \mathrm{L}$ was the best dose against the suppression and control of the potato late blight under the field conditions. Azoxystrobin which is a new chemistry fungicide found to be the best chemical treatment against the late blight of potato followed by the copper oxychloride-based fungicides Cobox which also showed best response.
Aliette, Antracol, Tebuconazole and Mancozed were also played a role to minimize the disease yet their higher concentration was even not so effective to combat the disease. Mancozeb was not found to be effective to control the disease as compared to the other three fungicides. Azoxystrobin at the rate of $5 \mathrm{~g} / \mathrm{L}$ gave best control against all the tested varieties to control the disease severity with 2.33, 2.73 and 3.33 on Desiree, Santee and Cardinal respectively. Similarly, copper oxychloride gave best response after the azoxystrobin at $5 \mathrm{~g} / \mathrm{L}$ with a control 2.91, 3.67 and 3.99 on Desiree, Santee and Cardinal respectively (Fig 1, 2, $3,4,5,6)$.

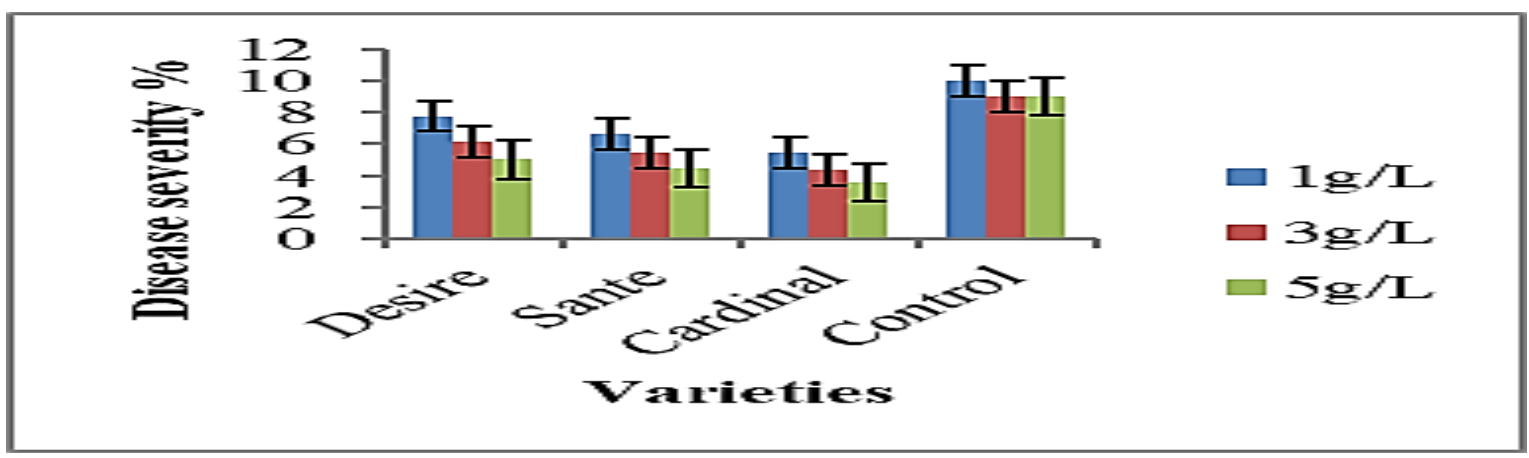

Figure 1. Disease severity $\%$ recorded after the application of aliette at the rate of different doses viz., 25,50 and $75 \mathrm{~g} / \mathrm{l}$.

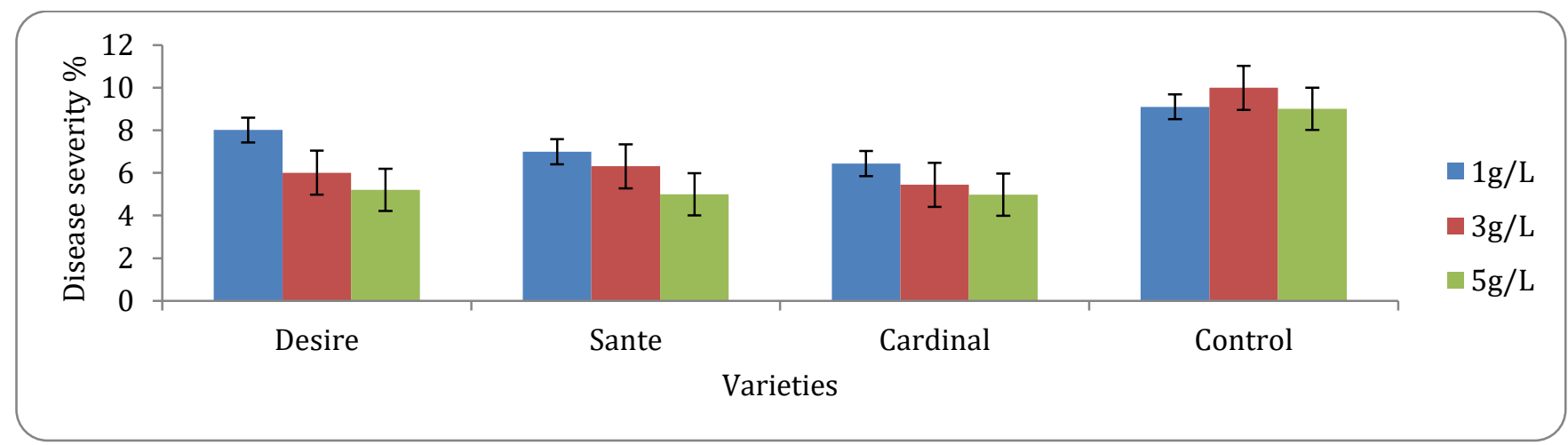

Figure 2. Disease severity \% recorded after the application of antracol at the rate of different doses viz., 25,50 and $75 \mathrm{~g} / \mathrm{l}$. 


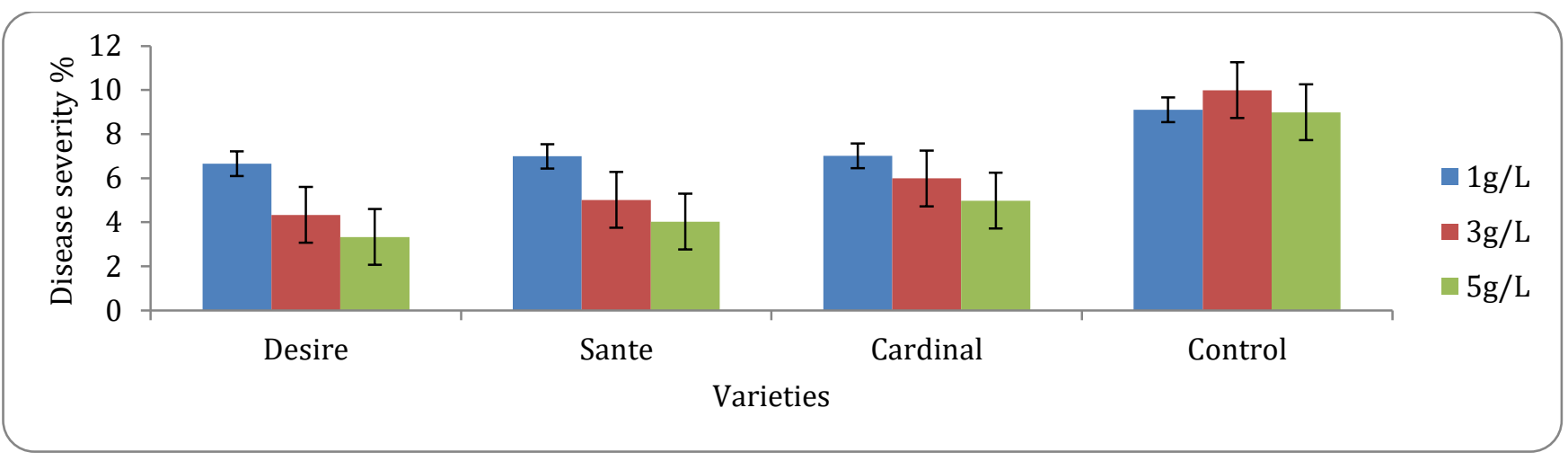

Figure 3. Disease severity \% recorded after the application of tebuconazole at the rate of different doses viz., 25, 50 and $75 \mathrm{~g} / \mathrm{l}$.

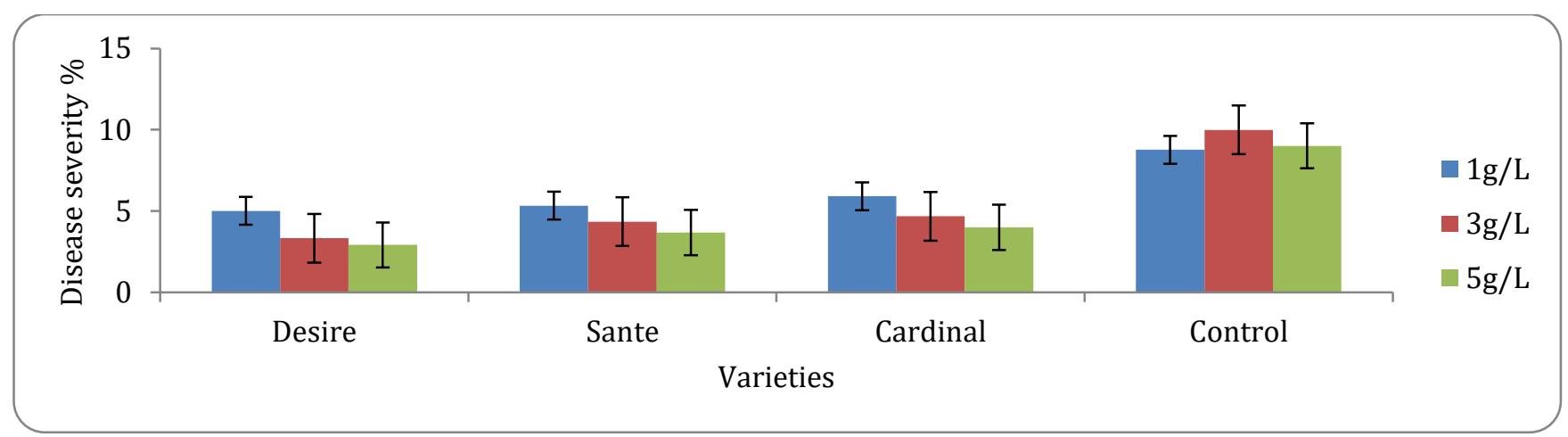

Figure 4. Disease severity \% recorded after the application of copper oxychloride at the rate of different doses viz., 25,50 and $75 \mathrm{~g} / \mathrm{l}$.

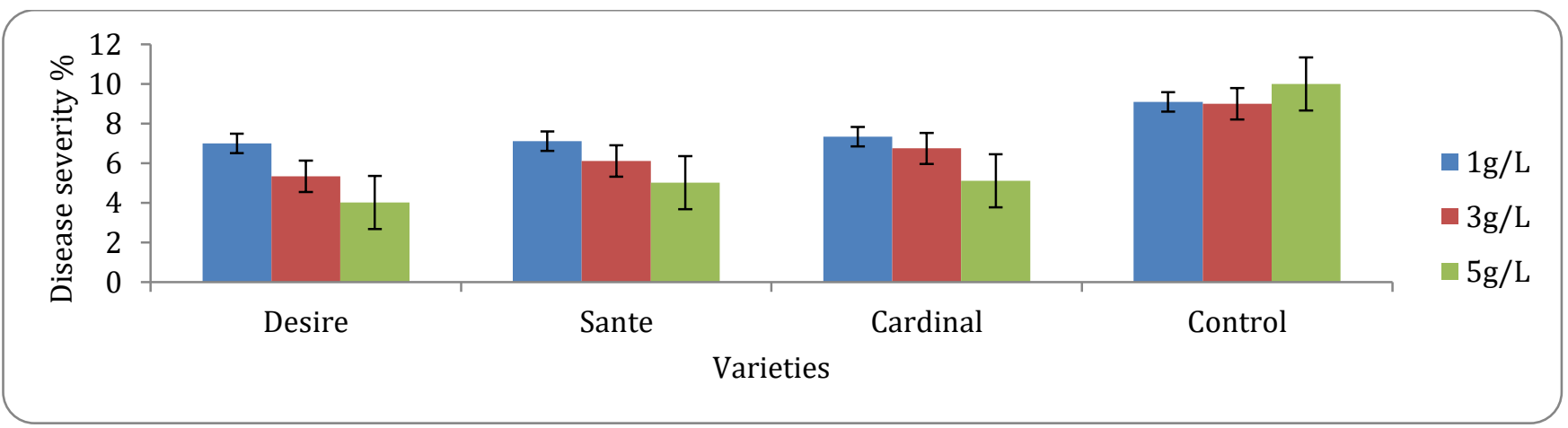

Figure 5. Disease severity \% recorded after the application of mancozeb at the rate of different doses viz., 25,50 and $75 \mathrm{~g} / \mathrm{l}$.

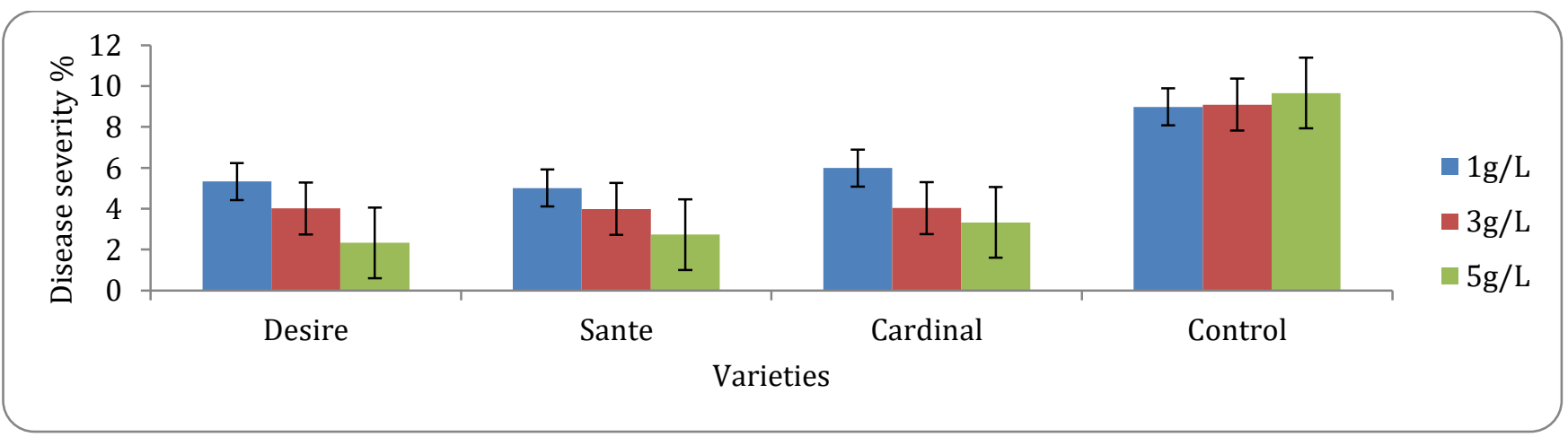

Figure 6. Disease severity \% recorded after the application of azoxystrobin at the rate of different doses viz., 25,50 and $75 \mathrm{~g} / 1$. 
In the case of botanical oils, mint and neem oils at the various concentrations showed varying response for the control of potato late blight under field conditions. Maximum control was obtained with mint oil with 2.99, 3.02 and 3.01 at $75 \mathrm{~g} / \mathrm{L}$ followed by Neem oil with 3.33, 4.01 and 3.61 with $75 \mathrm{~g} / \mathrm{L}$ concentration (Fig 7, 8, 9).

\section{Discussion}

The late blight fungus attacks potato leaves, stem and tubers. Asexually produced spores (sporangia) spread through the crop by wind and rain splash and the disease progresses very rapidly when temperature exceed $10^{\circ} \mathrm{C}$ and relative humidity is over $75 \%$ for 2 days or more. At temperature above about $15^{\circ} \mathrm{C}$, spores can infect leaves directly but at lower temperature, the spores germinate to produce 10-12 motile zoospores, each of which can swim in water films and infect the leaves. The fungus over winter as mycelium in potato tubers infected during the previous season by spores that were washed through the soil. As the temperature increase in the spring and the infected tuber begins to grow, the mycelium within it grows to produce an infected plant on which spore-bearing lesions develop. These spores infect newly planted potato crops and the disease cycle begins again (Ghorbani et al., 2004; Peart et al., 1994; Spits and Schepers, 2001; Trehan et al., 1995).

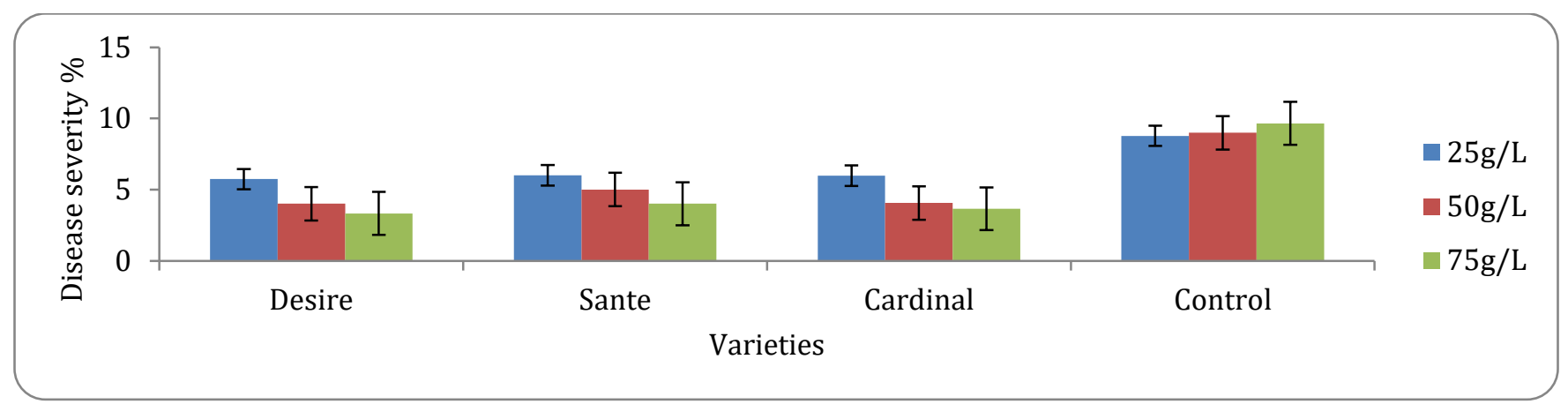

Figure 7. Disease severity \% recorded after the application of neem oil at the rate of different doses viz., 25, 50 and $75 \mathrm{~g} / \mathrm{l}$.

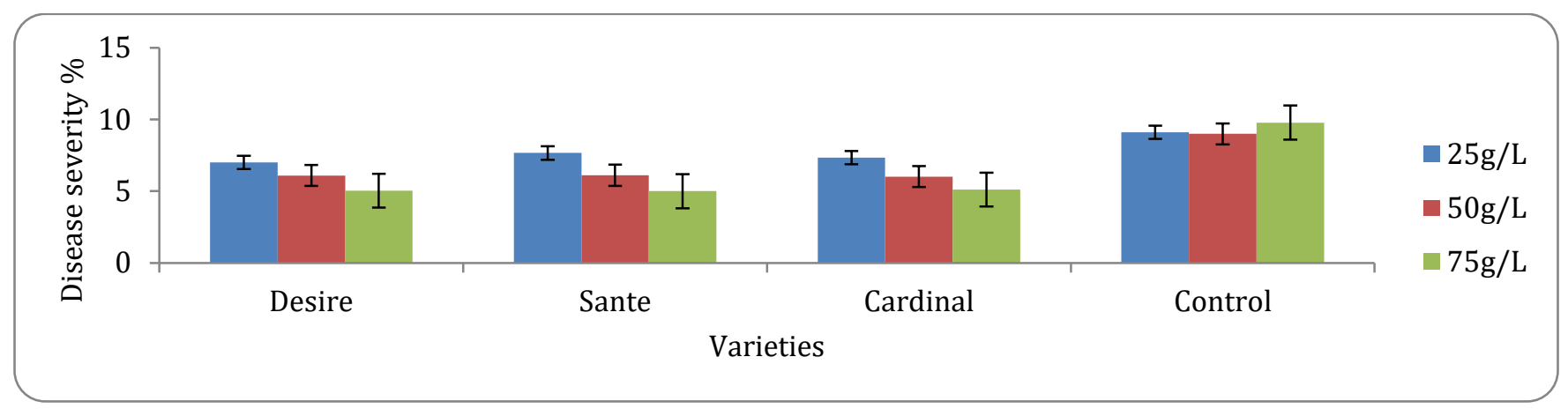

Figure 8. Disease severity \% recorded after the application of clove oil at the rate of different doses viz., 25,50 and $75 \mathrm{~g} / \mathrm{l}$.

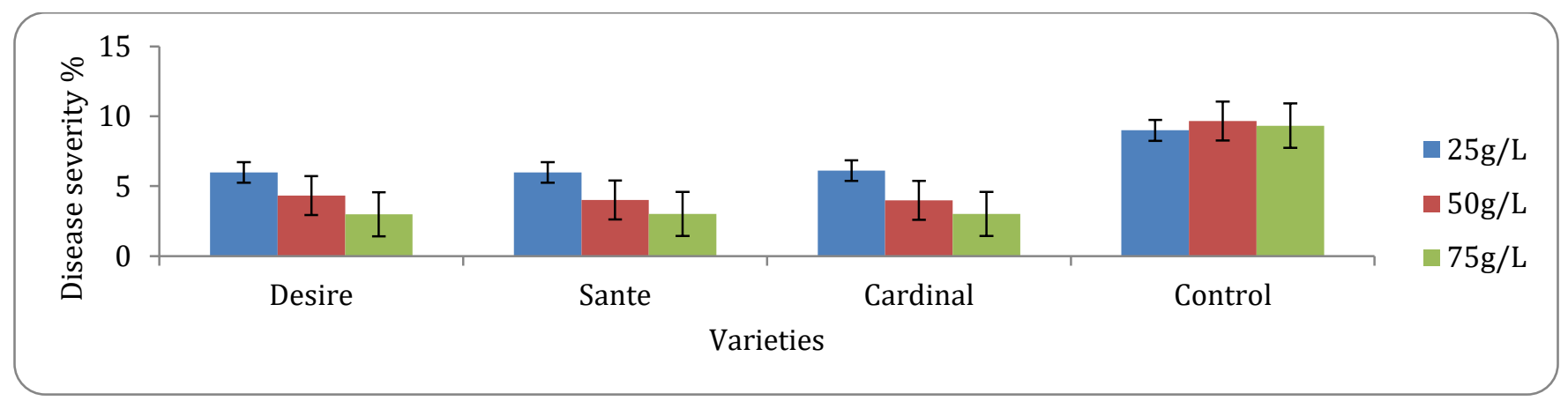

Figure 9. Disease severity \% recorded after the application of mint oil at the rate of different doses viz., 25, 50 and $75 \mathrm{~g} / \mathrm{l}$. 
DMRT test for the data of germplasm resistance indicated significant $(P<0.05)$ variation among all germplasm to late blight. Disease incidence and severity data of 68 potato varieties against potato late blight at different locations viz. Okara and Sahiwal showed statistically significant difference in mean disease incidence and severity at $5 \%$ level of significance. Out of 68 genotypes/varieties at Okara, the incidence showed 16 varieties as susceptible, 20 varieties as moderately resistant, five varieties as highly susceptible and 27 varieties as moderately susceptible. On the other hand, the maximum disease incidence at Okara was recorded at Desiree and Cardinal (90\%) followed by Santee (83.33\%), whereas minimum disease incidence was noted on SH-5 (26.67\%). Similarly, at Sahiwal area 16 genotypes were found susceptible, 36 as moderately susceptible, six varieties as highly susceptible and 10 varieties as moderately resistant. Maximum mean disease incidence at Sahiwal was noted on Cardinal and Desiree (91.67\%) and Karoda and Santee (90\%) while minimum disease incidence was noted $33.33 \%$ on SH 216 A, N13, FD 1-3 and FD 1-10. The disease severity data at Okara showed 34 genotypes as susceptible, 10 genotypes as moderately susceptible, 14 genotypes as moderately resistant and 10 genotypes as highly susceptible. The maximum mean disease severity 9.48 was noted on Cardinal, followed by Desiree 9.11 and Karoda 9.0 with the area under disease progress curve $28.55,27.33$ and 26.00 respectively while minimum disease severity was recorded 3.91 on Multa and 4.41 on FD 56-1 with the area under disease progress curve 11.33 and 13.33 respectively (Table 3). Similar type of research was also done by Cristmzio and Festa (1999) who studied the resistance of ten potato cultivars (Agria, Ajax, Desiree Liscta. Kennebec, Majestic, Monalisa, Prima, Spunta and Tonda di Berlino) to Phytophthora nfestans 8 fungal strains.

Fungicides and botanical oils tested against late blight of potato significantly reduced the disease severity as compared to control at all the tested concentrations. The results showed that $5 \mathrm{~g} / \mathrm{L}$ was the best dose against the suppression and control of the potato late blight under the field conditions. Azoxystrobin which is a new chemistry fungicide found to be the best chemical treatment against the late blight of potato followed by the copper oxychloride-based fungicides Cobox which also showed best response. Aliette, Antracol, Tebuconazole and Mancozed were also played a role to minimize the disease yet their higher concentration was even not so effective to combat the disease. Mancozeb was not found to be effective to control the disease as compared to the other three fungicides. Azoxystrobin at the rate of $5 \mathrm{gm} / \mathrm{L}$ gave best control against all the tested varieties to control the disease severity with $2.33,2.73$ and 3.33 on Desiree, Santee and Cardinal respectively. Similarly, Copper oxychloride gave best response after the azoxystrobin at $5 \mathrm{~g} / \mathrm{L}$ with a control 2.91, 3.67 and 3.99 on Desiree, Santee and Cardinal respectively (Fig $1,2,3,4,5,6)$. In the case of botanical oils mint and neem oils at various concentrations showed varying response for the control of potato late blight under field conditions. Maximum control was obtained with mint oil with 2.99, 3.02 and 3.01 at $75 \mathrm{~g} / \mathrm{L}$ followed by Neem oil with 3.33, 4.01 and 3.61 with $75 \mathrm{~g} / \mathrm{L}$ concentration. Our results are also in line with Speiser et al. (2006), Dowley and Sullivan (1994), Olanya et al. (2006) and Razukas et al. (2007) who described the potential of copper-based fungicides for the management of late blight of potato in Europe at different locations in different capacity. All the researchers showed that first the copper-based fungicides and the mixture of few new chemistries are found to be the most attractive one for the control of the devastating disease. The combination of Emisan- 6 with Blue Copper-50 and Streptocycline was found to be the most effective treatment. The reduction in disease intensity was coupled with an increase in yield for all the fungicide treatments. Although the climatic conditions were same at both locations but still the difference in severity of disease was observed. This might be due to the multiple factors, the soil condition, sub soil factors and pre disposing factors.

Conclusion: No variety was found to be resistant against this disease due to multiple factors. Fungicides are an option for the management of the disease to some extent.

Authors' contribution: AR, ARS and SAHN conceived the idea and conducted the research trials; ARS, UDU and HUKS wrote the manuscript; AR, SAHN performed the statistical analysis; AR, SAHN, MRB and HUKS reviewed and edited the manuscript.

Conflict of Interest: It is declared that there is no conflict of interest among the authors for the publication of said article in this prestigious journal and this article 
is submitted by the consent of all authors.

\section{REFERENCES}

Cristmzio, G., Festa, A., 1999. Testing cultivar resistance to Phytophthora Infestans. Potato Research 42, 101-105.

Dalsgaard, A., Pedersen, F.K., 1996. Shirlan, a new fungicide against potato late blight, 13th Danish Plant Protection Conference Pests and Disease Planteavlsforsog, pp. 65-75.

Dowley, L.J., Sullivan, 0., 1994. The effect of phenylamide/mancozeb mixtures on the control of late blight of potatoes. International Journal of Agriculture Food Science \& Technology 34, 38-43.

FAO, 2017. Status of potato in Pakistan. Food and Agriculture Organization.

Fry, W.E., Goodwin, S.B., Dyer, A., Mutuszak, T., Drenth, J.M., Tooley, A., Sujkowski, L.S., Koh, Y.J., Cohen, B.A., Spielman, L.J., Deahl, K.L., Sandlan, K.P., 1993. Historical and recent migration of Phytophthora infestans chronology, pathway and implications. Plant Disease 77, 653-661.

Ghorbani, R., Wilcockson, S.J., Giotis, C., Leifert, C., 2004. Potato late blight management in organic agriculture. Pest Management Journal 9, 11-15.

Hall, C.E., White, E.M., Cooke, L.R., Little, G., 2004. Potato varieties for organic production in Northern lreland., Organic forming science practice profitable livestock and cropping Proceeding. UGS/AAB/COR Conf, Newport, Shropshire UK,, pp. 236-239.

Kelman, A.H.E., 1984. Post harvest pathology of fruits and vegetables. Agricultural Expo Stn Bull, University of California, pp. 1-3.

Olanya, O.M., Ojiambo, P.S., Nyankanga, R.O., 2006. Dynamics of development of late blight (Phytophthora infestans) in potato, and comparative resistance of cultivars in the highland tropics. Canadian Journal of Plant Pathology 28, 84-94.

Peart, R.M., Papajorgji, P., Weingartner, P., Watson, D.G., Zazueta, F.S., Harrison, T.V., 1994. Potato late blight expert system written with DBASb IV. Computers in Agriculture, Proceedings of the 5th International Conference Orlando, Florida, USA, pp. 353-356.

Razukas, A., Jundulas, J., Asakaviciute, R., 2007. Potato cultivars susceptibility to potato late blight (Phytophthora infestans). Applied Ecology and Environmental Research 6, 95-106.

Shutong, W., Tongle, H.U., Fengqiao, Z., Forrer, H.R., Keqian, C.A.O., 2007. Screening for plant extracts to control potato late blight. Frontiers of Agriculture in China 1, 43-46.

Speiser, B., Tamm, L., Amsler, T., Lambion, J., Bertrand, C., Hermansen, A., Ruissen, M.A., Haaland, P., Zarb, J., Santos, J., Shotton, P., Wilcockson, S., Juntharathep, P., Ghorbani, R., Leifert, C., 2006. Improvement of late blight management in organic potato production systems in Europe: field tests with more resistant potato varieties and copper based fungicides. Biological Agriculture \& Horticulture 23, 393-412.

Spits, H.G., Schepers, H.T.A.M., 2001. Phytophthora infestans: protection of the growing point of potato plants by using fungicides. PPO Bulletin Akkerbouw 1, 2-4.

Steel, R.G.D., Torrie, J.H., Dicky, D.A., 1980. Principles and Procedures of Statistics: A Biometrical Approach, 3rd Edition ed. McGraw Hill Book Co., New York.

Trehan, S.P., Sharma, K.K., Grewal, J.S., 1995. Effect of soil and foliar application of micronutrients and fungicides on late blight (Phytophthora infestans) and tuber yield of potato (Solanwn wbaosum). Indian Journal of Agricultural Science 65, 836-841. 